\title{
Capecitabine and temozolomide combination for treatment of high-grade, well-differentiated neuroendocrine tumour and poorly-differentiated neuroendocrine carcinoma retrospective analysis
}

\author{
Wojciech Rogowski ${ }^{1,2}$, Ewa Wachuła ${ }^{3}$, Anita Gorzelak ${ }^{3}$, Aneta Lebiedzińska ${ }^{2,4}$, Violeta Sulżyc-Bielicka ${ }^{5}$, \\ Ewa Iżycka-Swieszewska ${ }^{6}$, Jakub Żołnierek ${ }^{1,7}$, Beata Kos-Kudła ${ }^{3}$ \\ ${ }^{1}$ Magodent sp. z o.o, Warsaw, Poland \\ ${ }^{2}$ University of Warmia and Mazury, Olsztyn, Poland \\ ${ }^{3}$ Medical University of Silesia, Katowice, Poland \\ ${ }^{4}$ Warmia and Mazury Oncology Centre, Olsztyn, Poland \\ ${ }^{5}$ Pomeranian Medical University, Szczecin, Poland \\ ${ }^{6}$ Medical University of Gdansk, Gdansk, Poland \\ ${ }^{7}$ Memorial Maria Sklodowska-Curie Oncology Institute, Warsaw, Poland
}

\begin{abstract}
Introduction: Many retrospective studies have confirmed that capecitabine combined with temozolomide is effective in neuroendocrine neoplasms. Most of the studies focused on grade 1 and grade 2 neuroendocrine tumours, mainly of pancreatic origin. There are limited data regarding the efficacy capecitabine with temozolomide in grade 3 neuroendocrine tumours. The new World Health Organisation 2017 classification distinguished well-differentiated grade 3 neuroendocrine tumours from poorly differentiated grade 3 neuroendocrine carcinomas. Treatment options for grade 3 neuroendocrine neoplasms are limited, and the overall prognosis is better in the subgroup of patients with grade 3 neuroendocrine tumours.

Material and methods: It was a retrospective study in the population of patients with diagnosed grade 3 neuroendocrine neoplasms of different origin treated with capecitabine and temozolomide. Data on clinical and demographic characteristics of the population were collected from four Polish clinical centres. This study aimed to evaluate response and survival parameters and compare outcomes of treatment of neuroendocrine tumours and carcinomas.

Results: The study included 32 patients with grade 3 neuroendocrine tumours treated with capecitabine and temozolomide. The disease control rate was twice as high in the group of patient with neuroendocrine tumours in comparison to carcinomas (70 vs. 30\%). The progression-free survival for patients with neuroendocrine tumours was 15.3 months (95\% CI: 3.9-30.4), and for patients with neuroendocrine carcinomas it was 3.3 months (95\% CI: 2.5-7.1). Median overall survival was 22 months (95\% CI: 11.8-22.0) and 4.6 months (95\% CI: 2.2-5.9) for patients with tumours and carcinomas, respectively. The treatment regimen was generally well tolerated.

Conclusions: The combination of capecitabine and temozolomide is an effective treatment for patients with grade 3 neuroendocrine tumours with Ki-67 index ranging between 20 and 54\%. The treatment did not overcome the aggressive character of neuroendocrine carcinomas and resulted in low response and survival outcomes in comparison to those achieved in tumour therapy. (Endokrynol Pol 2019; 70 (4): 313-317)
\end{abstract}

Key words: capecitabine; grade 3 neuroendocrine neoplasms; neuroendocrine carcinoma; neuroendocrine tumour; temozolomide

\section{Introduction}

The grading system for neuroendocrine tumours is based on the Ki-67 index and mitotic rate [1, 2]. A few per cent of all gastroenteropancreatic neuroendocrine neoplasms (NEN) have Ki-67 labelling index $>20 \%$ [3]. In the 2017 World Health Organisation classification of neuroendocrine neoplasms they were divided into low-to-intermediate grade 1 (G1) and 2 (G2) NETs, and grade 3 (G3) neoplasms [1, 2]. In the high-grade category there are neoplasms with Ki-67 $>20 \%$ but containing well-differentiated and poorly-differentiated neoplasms [4]. Poorly-differentiated G3 NEC has $>20$ mitoses/10 high-power fields (HPF) and/or Ki-67 labelling index usually $\geq 55 \%$. Well-differentiated G3 NET has Ki-67 usually between 20 and 54\% $[1,2,5]$. This separation has clinical significance. G3 NETs and NECs differ considerably concerning their biological behaviour and patients' outcome. The largest retrospective study on gastrointestinal NEN G3 - the so-called Nordic NEC trial - reported a lower response rate to platinum-based chemotherapy in patients with NETs 
compared to patients with high proliferative activity exceeding Ki-67 55\% (15\% vs. 42\%; p < 0.01). Overall prognosis was superior in the subgroup of patients with Ki-67 < 55\% [5]. Prognosis of patients with G3 NETs is worse in comparison to patients with a lower grade NET, but favourable compared to NECs.

Treatment options for G3 NENs are limited. Retrospective data suggest that commonly applied first-line chemotherapy protocols including cisplatin or carboplatin in combination with etoposide are less effective in G3 NET than in G3 NEC. According to the ENETS guidelines, therapeutic alternatives comprise temozolomide-based chemotherapy and peptide receptor radionucleotide therapy in somatostatin receptor-positive tumours [6]. Specific trials for G3 NENs are necessary, and new therapies are urgently needed [7].

Combination of capecitabine and temozolomide (CAPTEM) is one of the systemic treatments used mainly in G1 and G2 pancreatic NETs, and to a lesser extent in gastroenetropatic NETs $[8,9]$. There is no evidence from a randomised clinical trial on CAPTEM efficacy and safety. Data regarding the use of CAPTEM in G3 NENs is minimal, and neoplasms with Ki-67 > 20\% into NETs and NECs were not differentiated in the literature reporting the use of CAPTEM [9-12]. Recently sunitinib was shown to be as effective for G3 NETs as for G1/2 NETs [13].

Here, we present a retrospective analysis of clinical experience in patients with advanced stage G3 NENs, who were treated with CAPTEM.

\section{Material and methods}

\section{Patient characteristics}

Data of patients with G3 neuroendocrine neoplasms with Ki-67 proliferating index $>20$ and treated with at least one cycle of CAPTEM chemotherapy were collected from four Polish clinical centres from Katowice, Olsztyn, Szczecin, and Warsaw. There were no exclusion criteria to reflect the real-life practice of treatment of G3 NENs. Records submitted by treating physicians were checked and reviewed for eventual discrepancies and to obtain supplementary information. Background characteristics investigated included age, gender, tumour origin, grade, hormonal activity, and type of previous and concomitant treatment. Start and stop dates of CAPTEM treatment, type and severity of toxicities, response to treatment, date of progression, and date of death were recorded. Adverse events were graded according to the Common Toxicity Criteria of Adverse Events (CTCAE) version 4.0 [14].

Patients received capecitabine at $600 \mathrm{mg} / \mathrm{m}^{2}$ orally twice daily on days 1-14 (maximum $1000 \mathrm{mg}$ orally twice daily) and temozolomide $150-200 \mathrm{mg} / \mathrm{m}^{2}$ once daily or divided into two doses daily, on days 10-14 of a 28-day cycle. Dose modifications and dosing intervals were at the discretion of the treating physician.

Tumour response was routinely evaluated according to the response evaluation criteria in solid tumours (RECIST) [15]. Based on the Ki67 proliferative index tumours were divided according to the WHO 2017 Classification [1] to compare survival outcomes of CAPTEM treatment. The observation period for each patient started with initiation of CAPTEM treatment. The follow-up period for this analysis ended on January $15^{\text {th }} 2019$.

\section{Statistical analysis}

Descriptive statistical methods were used to summarise clinical characteristics. Progression-free survival (PFS) was defined as the time from the start of CAPTEM treatment to tumour progression or death. Overall survival (OS) was defined as the time from the start of CAPTEM treatment to death. Time-to-event data were analysed using Kaplan-Meier estimates. The log-rank test was used to compare survival curves, and the effect of possible prognostic value of the Ki-67 index was calculated by Cox proportional hazards model. The data were analysed using MedCalc, version 18.11.3.

\section{Results}

A total of 32 patients with G3 NENs and treated with CAPTEM were identified. All patients had started CAPTEM therapy during the last five years (between 2013 and 2018), and follow-up ended at January $15^{\text {th }}$, 2019. Patient characteristics are described in Table I. The mean $( \pm S D)$ age was $55.0( \pm 15.8)$ years (range $27-78$ years). Thirty-one patients had non-functional tumours and one patient had a secreting tumour (insulinoma pancreatic NET). The median Ki-67 index was 52\% (range 20-95\%). Twenty patients were diagnosed with well-differentiated G3 NETs, with Ki-67 ranging from 20 to 54 . Twelve patients had neoplasms with Ki-67 proliferation index $\geq 55 \%$ and diagnosis of G3 NEC. CAPTEM was used in the first-line in almost every third patient and in half of the patients in the second-line. The maximal number of previous lines of therapy before CAPTEM was four. The most common previous treatment was etoposide and cisplatin chemotherapy.

Table I. Baseline characteristics and demographics of patients

\begin{tabular}{lc}
\hline Characteristic & Population (n = 32) \\
\hline Age, mean \pm SD, range, years & $15(46.0 \pm 15.8,27-78$ \\
\hline Male gender, $\mathbf{n}(\%)$ & $1(3.1)$ \\
\hline Origin of tumours, $\mathbf{n}(\%)$ & $5(15.6)$ \\
\hline Appendix & $5(15.6)$ \\
\hline Colon & $13(40,6)$ \\
\hline Gastric & $1(3.1)$ \\
\hline Pancreas & $2(6.2)$ \\
\hline Rectum & $5(15.6)$ \\
\hline Retroperitoneal & \\
\hline Small bowel & $31(96.9)$ \\
\hline Tumour functionality, n (\%) & $1(3.1)$ \\
\hline Non-functioning & $20(62.5)$ \\
\hline Insulinoma & $12(37.5)$ \\
\hline Ki-67 index, mean \pm SD, range, \% & $25.9,20-95$ \\
\hline Tumour grade* $\mathbf{n}(\%)$ & \\
\hline G3 NET (Ki-67 $<55 \%), \mathbf{n}(\%)$ & \\
\hline G3 NEC (Ki-67 $\geq 55 \%), \mathbf{n}(\%)$ & \\
\hline & \\
\hline
\end{tabular}


Table I. Baseline characteristics and demographics of patients

\begin{tabular}{ll}
\hline Characteristic & Population $(\mathbf{n}=\mathbf{3 2})$ \\
\hline Treatment line, $\mathbf{n}(\%)$ & $10(31.3)$ \\
\hline $1^{\text {st line }}$ & $16(50.0)$ \\
\hline $2^{\text {nd }}$ line & $8(25.0)$ \\
\hline$\geq 3^{\text {rd line }}$ & $9(28.1)$ \\
\hline CAPTEM + SSA & $14(43.8)$ \\
\hline Previous treatment, $\mathbf{n}(\%)$ & $3(9.3)$ \\
\hline ETO + PLA & $2(6.2)$ \\
\hline ETO + PLA + SSA & $2(6.2)$ \\
\hline CAV & $1(3.1)$ \\
\hline PRRT & $1(3.1)$ \\
\hline PLA-Vinorelbine & $1(3.1)$ \\
\hline DOXO+ DDP & $2(6.2)$ \\
\hline DTIC+FFU & $2(6.2)$ \\
\hline PRRT & $2(6.2)$ \\
\hline Radiotherapy & $2(6.2)$ \\
\hline Streptozotocin & $1(3.1)$ \\
\hline Streptozotocin + 5FU & \\
\hline Sunitinib & \\
\hline CAV & \\
\hline
\end{tabular}

CAV — cyclophosphamide, doxorubicin, vincristine; ETO + PLA — etoposide, cisplatin; DOXO — doxorubicin; DDP — diamine-dichloro-platinum;

DTIC — dacarbazine; FFU — FOLFIRI- irinotecan, folinic acid, 5-fluorouracil;

PRRT — peptide receptor radionuclide therapy; $5 \mathrm{FU}-5$-fluorouracil;

SSA — somatostatin analogues; *According to 2017 WHO classification [1]
CAPTEM was used in combination with a somatostatin analogue in nine patients.

The median $( \pm S D)$ time duration of treatment was 5.4 months (range 1.0-30.4) months. Table II summarises the best response to treatment. The disease control rate was twice as high in the group of patients with NETs than in the G3 NEC group.

Median PFS for all patients was 7.1 months (95\% CI: 3.27-15.3). Median PFS was significantly higher in the G3 NET group (15.3 months, 95\% CI: 3.9-30.4) than in the G3 NEC group (3.3 months, 95\% CI: 2.5-7.1) (hazard ratio 5.5, 95\% CI: 2.0-15.0, p = 0.0009) (Fig. 1).

Seventeen $(53.1 \%)$ patients died. Median OS was 15.6 months (95\% CI: 8-22). Patients with NET-G3 had better overall survival (22 months; 95\% CI: 11.8-22.0) than patients with NEC-G3 (4.6 months; 95\% CI: 2.2-5.9) (Fig. 1). The difference was statistically significant (hazard ratio 4.0 95\% CI: 1.2-13.0, $\mathrm{p}=0.0178$ ).

The CAPTEM regimen was generally well tolerated. Grade 3 and 4 toxicities included thrombocytopaenia in five patients, neutropaenia in three patients, diarrhoea and hand-foot syndrome in two patients each, and fatigue in one patient. The most common grade 1 and 2 toxicities were thrombocytopaenia, fatigue, neutropaenia, diarrhoea, and hand-foot syndrome. There were no opportunistic infections or adverse event-related deaths. There were two hospitalisations

Table II. Response to treatment

\begin{tabular}{lccc}
\hline Response to treatment & $\begin{array}{c}\text { All patients } \\
(\mathbf{n}=\mathbf{3 2})\end{array}$ & $\begin{array}{c}\text { G3 NET (Ki-67 <55\%) } \\
(\mathbf{n}=\mathbf{2 0})\end{array}$ & $\begin{array}{c}\text { G3 NEC (Ki-67 } \geq \mathbf{5 5 \% )} \\
(\mathbf{n}=\mathbf{1 2})\end{array}$ \\
\hline Progressive disease, $\mathrm{n}(\%)$ & $14(43.8 \%)$ & $6(30.0 \%)$ & $8(66.7 \%)$ \\
\hline Stable disease, $\mathrm{n}(\%)$ & $7(21.9 \%)$ & $4(20.0 \%)$ & $3(25.0 \%)$ \\
\hline Partial response, $\mathrm{n}(\%)$ & $11(34.4 \%)$ & $10(50.0 \%)$ & $1(8.3 \%)$ \\
\hline Disease control rate $(\%)$ & $56 \%$ & $70 \%$ & $33 \%$ \\
\hline
\end{tabular}
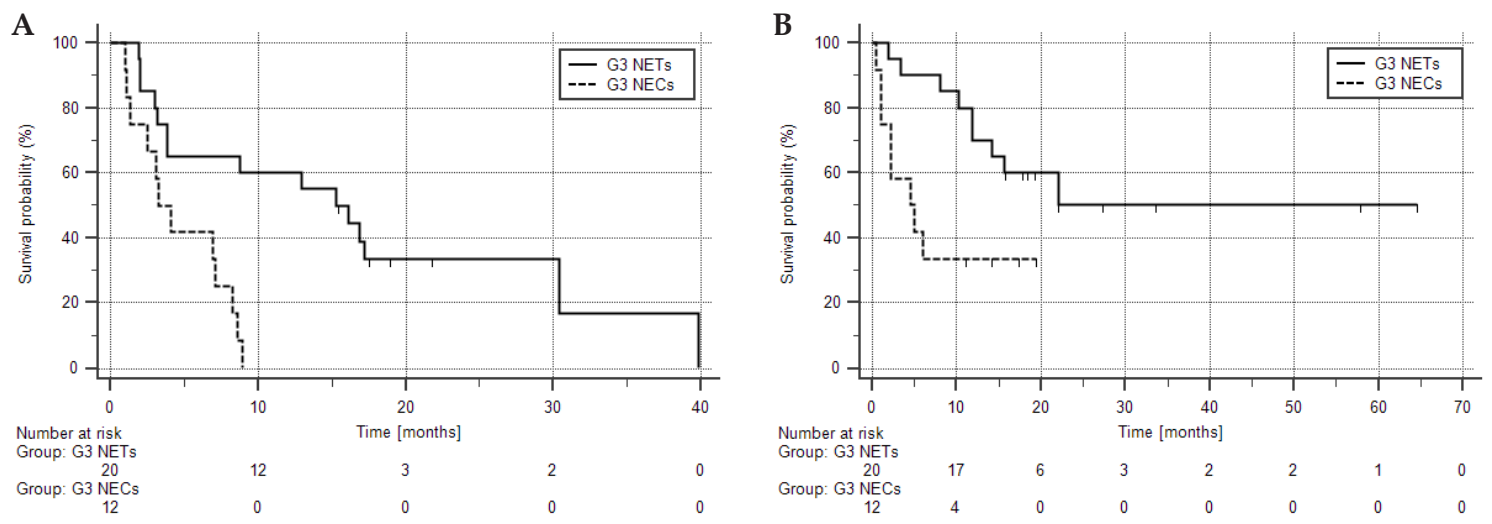

Figure 1. Survival outcomes of treatment with capecitabine and temozolomide: A. Progression-free survival. B. Overall survival in patients diagnosed with grade 3 neuroendocrine tumours (G3 NETs) and carcinomas (G3 NECs) 
Table III. Treatment toxicity

\begin{tabular}{lcc}
\hline Adverse event & \multicolumn{2}{c}{ Number of patients with } \\
\cline { 2 - 3 } & Grade 1-2 & Grade 3-4 \\
\hline Thrombocytopaenia & 12 & 5 \\
\hline Neutropaenia & 9 & 3 \\
\hline Fatigue & 11 & 1 \\
\hline Hand-foot syndrome & 8 & 2 \\
\hline Diarrhoea & 8 & 2 \\
\hline Nausea & 5 & 0 \\
\hline Mucositis & 3 & 0 \\
\hline Anaemia & 4 & 0 \\
\hline Infection & 6 & 0 \\
\hline Neuropathy & 3 & 0 \\
\hline Hyperglycaemia & 3 & 0 \\
\hline
\end{tabular}

due to haematological toxicities, which fully recovered. Treating physicians seven times decided to decrease dose or delay next treatment cycle due to adverse events. All reported adverse events are summarised in Table III.

\section{Discussion}

Treatment for G3 NETs and NECs is not standardised. The first study to focus on the treatment of G3 NETs was conducted in 1991. The aim of the study was to access the effectiveness of cisplatin and etoposide in patients with G3 NETs. The results achieved in small cell lung cancer treatment inspired authors to evaluate that regimen in NECs [16]. The study for the first time showed that poorly-differentiated NECs show a better response $(\mathrm{ORR}=67 \%)$ than well-differentiated NETs $(\mathrm{ORR}=7 \%)$ to platinum-based chemotherapy. The toxicity of the treatment regimen was not acceptable. Thus, subsequent studies aimed to control the toxicity by reducing the dose of etoposide, shortening the infusion time of cisplatin, and identifying predictors of high toxicity $[17,18]$. Attempts to add paclitaxel to carboplatin and etoposide were unsuccessful [19]. In G3 NECs platinum-based chemotherapy is recommended as the first-line therapy $[20,21]$. The NORDIC NEC retrospective analysis published in 2012 showed that G3 neoplasms represent a heterogeneous group, because patients with well-differentiated tumours and lower Ki-67 (20-55\%) show a poorer response to platinum-based chemotherapy than patients with Ki-67 > 55\% [5]. As a result, the WHO G3 category was subdivided into two subtypes: G3 NET (Ki-67 20-55\%) and G3 NEC (Ki-67 > 55\%) [1,2]. The biological behaviour of tumours with a Ki-67 index between 20 and $55 \%$ is less aggressive than that of tumours with a Ki-67 index above $55 \%$, thus response to the same treatment is expected to be different. The distinction between G3 NETs and G3 NECs is extremely important in determining treatment options for patients with G3 NENs.

The first randomised study reporting efficacy of cytotoxic therapy in NETs was by Moertel et al. [22]. It showed that streptozotocin-based therapies prolonged the survival time. Most of the other studies were studies without a control group; however, they resulted in widespread use of different alkylating agents and their combinations in the treatment of NETs [23]. Among them, a temozolomide-based regimen has emerged as a promising, well-tolerated, oral treatment. We are lacking an adequate prospective controlled study defining the role of temozolomide in the treatment of NETs. The first controlled study to evaluate the usefulness of CAPTEM was the study by the ECOG-ACRIN Cancer Research Group. It showed that median PFS was 22.7 months for CAPTEM vs. 14.4 months for TEM in patients with low- or intermediate-grade advanced pancreatic NETs [25]. There are many retrospective and single-arm reports about CAPTEM use mainly in G1/G2 NETs of the pancreas $[8,9,26,27]$, but data on G3 NENs is very limited. The confirmed efficacy of the CAPTEM regimen in G1/2 NETs encouraged attempts to treat G3 NETs in the second-line therapy [5] and in patients with Ki-67 >20\% [9-12]. In the literature we found descriptions of outcomes of CAPTEM therapy in 18 patients with Ki-67 > 20\% [9-12], and the treatment of NECs remains the domain of case reports [28].

Here we present a cohort of 32 patients with G3 NENs treated with CAPTEM regimen. To our knowledge, this is the first study documenting the efficacy and safety of the CAPTEM regimen in subgroups of patients with high Ki-67 index, divided into G3 NETs and NECs based on the WHO 2017 classification [1]. The median PFS for all patients of 7.1 months (95\% CI: 3.27-15.3) was comparable to the range from 3.4 to 6.0 months reported in the recent metanalysis by Lu et al. [29]. However, comparison of survival and response based on the Ki-67 level cut-off at 55\% showed that results of the treatment were better in G3 NETs than in NECs. It is in line with the recent results for sunitinib efficacy in the treatment of G3 NENs [30]. G3 NETs and NECs are considered as different entities [1]. NECs have an aggressive nature and a poor prognosis in comparisons to G3 NETs [5, 30]. CAPTEM treatment did not show to overcome aggressive character of NECs.

Our study has some significant limitations. Because G3 NENs are rare, the study population was small. In addition, as a retrospective study the nonrandomised patient population were constraints in the analysis. Accepting the limitations, our study presents unique data 
on the efficacy of CAPTEM in the treatment of G3 NENs, with their differentiation to NETs and NECs.

\section{Conclusions}

CAPTEM is an effective treatment for patients with G3 NETs. The treatment did not overcome the aggressive character of NECs and resulted in low response and survival outcomes in comparison to those achieved in tumour therapy.

\section{Acknowledgments}

The authors thank Ewelina Drelich and Marcin Balcerzak of Farenta for providing statistical and medical writing support. The authors declare no conflict of interest.

\section{References}

1. Klöppel G, Couvelard A, Hruban RH. Neoplasms of the neuroendocrine pancreas. WHO Classification of Tumours of Endocrine Organs. 4th ed. International Agency for Research on Cancer, Lyon 2017: 210-239.

2. Kos-Kudła B, Blicharz-Dorniak J, Strzelczyk J, et al. Consensus Conference, Polish Network of Neuroendocrine Tumours. Diagnostic and therapeutic guidelines for gastro-entero-pancreatic neuroendocrine neoplasms (recommended by the Polish Network of Neuroendocrine Tumours). Endokrynol Pol. 2013; 64(6): 418-443, doi: 10.5603/EP.2013.0028, indexed in Pubmed: 24431116.

3. Garcia-Carbonero R, Capdevila J, Crespo-Herrero G, et al. Incidence, patterns of care and prognostic factors for outcome of gastroenteropancreatic neuroendocrine tumors (GEP-NETs): results from the National Cancer Registry of Spain (RGETNE). Ann Oncol. 2010; 21(9): 1794-1803, doi: 10.1093/annonc/mdq022, indexed in Pubmed: 20139156.

4. Basturk O, Yang Z, Tang LH, et al. The high-grade (WHO G3) pancreatic neuroendocrine tumor category is morphologically and biologically heterogenous and includes both well differentiated and poorly differentiated neoplasms. Am J Surg Pathol. 2015; 39(5): 683-690, doi: 10.1097/PAS.0000000000000408, indexed in Pubmed: 25723112

5. Sorbye H, Welin S, Langer SW, et al. Predictive and prognostic factors for treatment and survival in 305 patients with advanced gastrointestinal neuroendocrine carcinoma (WHO G3): the NORDIC NEC study. Ann Oncol. 2013; 24(1): 152-160, doi: 10.1093/annonc/mds276, indexed in Pubmed: 22967994.

6. Garcia-Carbonero R, Sorbye H, Baudin E, et al. Vienna Consensus Conference participants. ENETS Consensus Guidelines for High-Grade Gastroenteropancreatic Neuroendocrine Tumors and Neuroendocrine Carcinomas. Neuroendocrinology. 2016; 103(2): 186-194, doi: 10.1159/000443172, indexed in Pubmed: 26731334.

7. Rinke A, Gress TM. Neuroendocrine Cancer, Therapeutic Strategies in G3 Cancers. Digestion. 2017; 95(2): 109-114, doi: 10.1159/000454761, indexed in Pubmed. 28161703.

8. Crespo G, Jiménez-Fonseca P, Custodio A, et al. Capecitabine and temozolomide in grade $1 / 2$ neuroendocrine tumors: a Spanish multicenter experience. Future Oncol. 2017; 13(7): 615-624, doi: 10.2217/fon-2016-0434, indexed in Pubmed: 27802780.

9. Cives M, Ghayouri M, Morse B, et al. Analysis of potential response predictors to capecitabine/temozolomide in metastatic pancreatic neuroendocrine tumors. Endocr Relat Cancer. 2016; 23(9): 759-767, doi: 10.1530/ERC-16-0147, indexed in Pubmed: 27552969.

10. Chauhan A, Farooqui Z, Murray LeA, et al. Capecitabine and Temozolomide in Neuroendocrine Tumor of Unknown Primary. J Oncol. 2018; 2018: 3519247, doi: 10.1155/2018/3519247, indexed in Pubmed: 29853889.

11. Owen DH, Alexander AJ, Konda B, et al. Combination therapy with capecitabine and temozolomide in patients with low and high grade neuroendocrine tumors, with an exploratory analysis of O-methylguanine DNA methyltransferase as a biomarker for response. Oncotarget. 2017; 8(61): 104046-104056, doi: 10.18632/oncotarget.22001, indexed in Pubmed: 29262620.

12. Ramirez RA, Beyer DT, Chauhan A, et al. The Role of Capecitabine/Temozolomide in Metastatic Neuroendocrine Tumors. Oncologist. 2016 21(6): 671-675, doi: 10.1634/theoncologist.2015-0470, indexed in Pubmed: 27226359.
13. Mizuno Y, Kudo A, Akashi T, et al. Sunitinib shrinks NET-G3 pancreatic neuroendocrine neoplasms. J Cancer Res Clin Oncol. 2018; 144(6) 1155-1163, doi: 10.1007/s00432-018-2636-2, indexed in Pubmed: 29602973.

14. National Cancer Institute: Protocol Development | Adverse Events/CTCAE. National Cancer Institute at the National Institutes of Health; c2018. Common Terminology Criteria for Adverse Events (CTCAE) v4.0. 2010 Jun 14. Nhttps://ctep.cancer.gov/protocolDevelopment/electronic applications/docs/CTCAE_4.03.xlsx (2019 Jan 29).

15. Eisenhauer EA, Therasse P, Bogaerts J, et al. New response evaluation criteria in solid tumours: revised RECIST guideline (version 1.1). Eur J Cancer. 2009; 45(2): 228-247, doi: 10.1016/j.ejca.2008.10.026, indexed in Pubmed: 19097774.

16. Moertel CG, Kvols LK, O'Connell MJ, et al. Treatment of neuroendocrine carcinomas with combined etoposide and cisplatin. Evidence of major therapeutic activity in the anaplastic variants of these neoplasms. Cancer. 1991; 68(2): 227-232, indexed in Pubmed: 1712661.

17. Mitry E, Baudin E, Ducreux M, et al. Treatment of poorly differentiated neuroendocrine tumours with etoposide and cisplatin. Br J Cancer. 1999; 81(8): 1351-1355, doi: 10.1038/sj.bjc.6690325, indexed in Pubmed: 10604732

18. Fjällskog ML, Granberg DP, Welin SL, et al. Treatment with cisplatin and etoposide in patients with neuroendocrine tumors. Cancer. 2001; 92(5): 1101-1107, indexed in Pubmed: 11571721.

19. Hainsworth JD, Spigel DR, Litchy S, et al. Phase II trial of paclitaxel, carboplatin, and etoposide in advanced poorly differentiated neuroendocrine carcinoma: a Minnie Pearl Cancer Research Network Study. J Clin Oncol. 2006; 24(22): 3548-3554, doi: 10.1200/JCO.2005.05.0575, indexed in Pubmed: 16877720 .

20. Pavel M, Baudin E, Couvelard A, et al. Barcelona Consensus Conference participants. ENETS Consensus Guidelines for the management of patients with liver and other distant metastases from neuroendocrine neoplasms of foregut, midgut, hindgut, and unknown primary. Neuroendocrinology. 2012; 95(2): 157-176, doi: 10.1159/000335597, indexed in Pubmed: 22262022

21. Delle Fave G, O'Toole D, Sundin A, et al. Vienna Consensus Conference participants. ENETS Consensus Guidelines Update for Gastroduodenal Neuroendocrine Neoplasms. Neuroendocrinology. 2016; 103(2): 119-124, doi: 10.1159/000443168, indexed in Pubmed: 26784901.

22. Moertel CG, Lefkopoulo M, Lipsitz S, et al Streptozocin-doxorubicin, streptozocin-fluorouracil or chlorozotocin in the treatment of advanced islet-cell carcinoma. N Engl J Med. 1992; 326(8): 519-523, doi: 10.1056/NEJM199202203260804, indexed in Pubmed: 1310159.

23. Okusaka T, Ueno H, Morizane C, et al. Cytotoxic chemotherapy for pancreatic neuroendocrine tumors. J Hepatobiliary Pancreat Sci. 2015 22(8): 628-633, doi: 10.1002/jhbp.257, indexed in Pubmed: 25940377.

24. U.S. National Library of Medicine. NCT01824875. Temozolomide With or Without Capecitabine in Treating Patients With Advanced Pancreatic Neuroendocrine Tumours. https://clinicaltrials.gov/ (2019 Feb 03).

25. Kunz $\mathrm{P}$ Catalano $\mathrm{P}$ Nimeiri $\mathrm{H}$, et al A randomized study of temozolomide or temozolomide and capecitabine in patients with advanced pancreatic neuroendocrine tumors: A trial of the ECOG-ACRIN Cancer Research Group (E2211). J Clin Oncol. 2018; 36(15(suppl)): 4004-4004 doi: 10.1200/jco.2018.36.15_suppl.4004.

26. Strosberg JR, Fine RL, Choi J, et al. First-line chemotherapy with capecitabine and temozolomide in patients with metastatic pancreatic endocrine carcinomas. Cancer. 2011; 117(2): 268-275, doi: 10.1002/cncr.25425, indexed in Pubmed: 20824724

27. Peixoto RD, Noonan KL, Pavlovich P, et al. Outcomes of patients treated with capecitabine and temozolamide for advanced pancreatic neuroendocrine tumors (PNETs) and non-PNETs. J Gastrointest Oncol. 2014; 5(4): 247-252, doi: 10.3978/j.issn.2078-6891.2014.019, indexed in Pubmed: 25083296.

28. Quinn AM, Chaturvedi A, Nonaka D. High-grade Neuroendocrine Carcinoma of the Lung With Carcinoid Morphology: A Study of 12 Cases. Am J Surg Pathol. 2017; 41(2): 263-270, doi: 10.1097/PAS.0000000000000767, indexed in Pubmed: 27879513.

29. Lu Y, Zhao Z, Wang Ji, et al. Safety and efficacy of combining capecitabine and temozolomide (CAPTEM) to treat advanced neuroendocrine neoplasms: A meta-analysis. Medicine (Baltimore). 2018; 97(41): e12784 doi: 10.1097/MD.0000000000012784, indexed in Pubmed: 30313101.

30. Mizuno Y, Kudo A, Akashi T, et al. Sunitinib shrinks NET-G3 pancreatic neuroendocrine neoplasms. J Cancer Res Clin Oncol 2018; 144(6): 1155-1163, doi: 10.1007/s00432-018-2636-2, indexed in Pubmed: 29602973.

31. Heetfeld M, Chougnet CN, Olsen $\mathrm{IH}$, et al other Knowledge Network members. Characteristics and treatment of patients with G3 gastroenteropancreatic neuroendocrine neoplasms. Endocr Relat Cancer. 2015; 22(4): 657-664, doi: 10.1530/ERC-15-0119, indexed in Pubmed: 26113608. 